\title{
REVIEW
}

\section{Current Imaging Mass Spectrometry for Metabolite Molecules}

\author{
Yuki Sugiura ${ }^{1,2 *}$ and Mitsutoshi Setou ${ }^{2,3}$ \\ ${ }^{1}$ Department of Bioscience and Biotechnology, Tokyo Institute of Technology, Yokohama, JAPAN \\ ${ }^{2}$ Mitsubishi Kagaku Institute of Life Sciences, Machida, TOKYO, JAPAN \\ ${ }^{3}$ Hamamatsu University School of Medicine, Department of Molecular Anatomy, Hamamatsu, JAPAN
}

\begin{abstract}
Matrix-assisted laser desorption/ionization (MALDI)-imaging mass spectrometry (IMS; also referred to as mass spectrometry imaging; MSI) enables the visualization of the distribution of a range of biomolecules that have varied structures in the cells and tissue sections. This emerging imaging technique was initially developed as a tool for protein imaging, but recently it is increasingly being used for the imaging of small organic molecules. IMS is an effective technique for the imaging of small metabolites, including endogenous metabolites such as lipids and exogenous drugs because of the following advantages: First, IMS does not require any specific labels or probes. Second, IMS is a non-targeted imaging method. Finally, the simultaneous imaging of many types of metabolite molecules is possible, and all these features are necessary for the assessment of metabolite localization. In this review, we discuss the capability of current IMS techniques for imaging small molecules, and introduce representative studies on imaging of endogenous and exogenous metabolites. In addition, the limitations and problems of the technique are also discussed, and reports of progress toward solving the problems with this technique are also introduced.
\end{abstract}

(Received December 1, 2008; Accepted January 13, 2009)

\section{Introduction}

Traditional matrix-assisted laser desorption/ionization mass spectrometry (MALDI-MS) can be applied to the analysis of a wide range of molecules with varied physical and chemical characteristics. In the biological and medical fields, MALDI-imaging mass spectrometry (IMS) can be used for the visualization of the distribution of large number of biomolecules in the cells and tissues, ranging from small metabolite molecules ${ }^{1)}{ }^{2)}$ to much larger proteins. ${ }^{3), 4)}$

This emerging imaging technique was initially developed as a tool for protein imaging. ${ }^{5)-7)}$ In fact, most of the reports concerning MALDI-IMS thus far describe the detection and imaging of proteins or peptides. On the other hand, research directed toward the detection and imaging of small organic molecules has recently been expanding. Figure 1 shows the result of a PubMed search (reviews were excluded) using "imaging mass spectrometry" as keywords. Reports are subdivided into groups according to the materials analyzed in the study, and the number of reports in each group is indicated. Notably, the number of reports regarding the IMS of small compounds has gradually increased, and occupies half of the works published in 2007. The hallmark of this trend is that a new IMS session entitled "imaging of small molecules" was established in the annual meeting of American Society for Mass Spectrometry in 2007. Note that another trend is apparent: reports describing development of data management procedure utilizing statisti-

* Correspondence to: Yuki Sugiura, Department of Bioscience and Biotechnology, Tokyo Institute of Technology, 4259 Nagatsuta-cho, Midori-ku, Yokohama 226-8501, JAPAN, e-mail: yuki.sgi@gmail.com

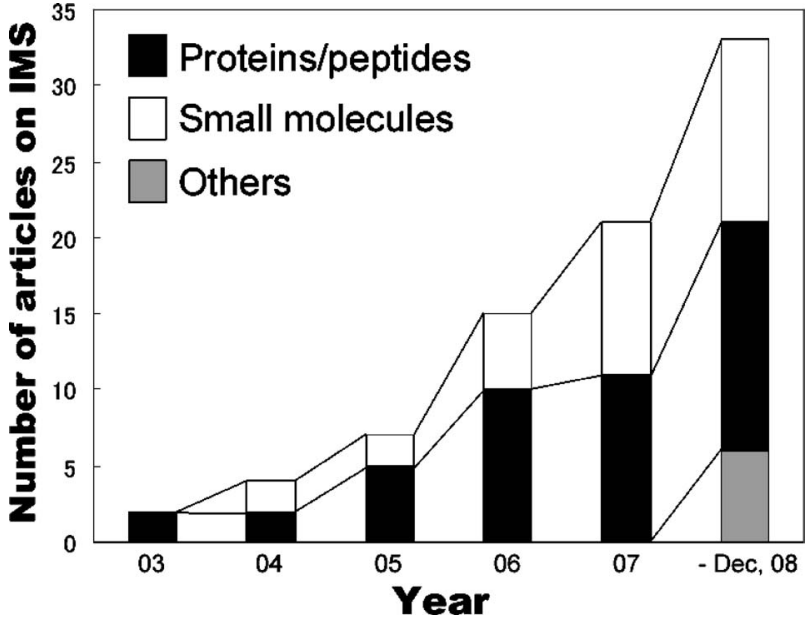

Fig. 1. Result of the PubMed search using "imaging mass spectrometry" as keywords.

cal analysis also increased in 2008 (labeled "other" in Fig. 1).

\section{Advantages of MALDI-IMS in Metabolite Imaging}

IMS (including both MALDI- and secondary ion mass spectrometry (SIMS)-based IMS) is an effective technique for imaging the distribution of small metabolites, in terms of metabolic intermediates, including lipids, amino acids, organic acids, and other small organic molecules. The emergence of IMS as a tool for metabolite imaging has much impact because we do not have an established technology for metabolite imaging, while localization of transcripts is visualized with oligonucleotide probe in situ hybridization, and localization of proteins is visualized using im- 
Table 1. Representative Molecular Imaging Methods in Tissues or Cells and Potential IMS Contrbution

\begin{tabular}{|c|c|c|}
\hline & Representative methods & Probe \\
\hline DNA & FISH (fluorescence in situ hybridization) & Oligo nucleotide probe \\
\hline RNA & in situ hybridization & Oligo nucleotide probe \\
\hline Protein & Immunohistochemistry, green fluorescent protein-fused protein & Antibody \\
\hline Metabolites (especially lipids) & Imaging mass spectrometry & - \\
\hline
\end{tabular}

Table 2. Imaging/Detection of Small Metabolite Molecules in Tissues by MALDI and Related Ionization Techniques

\begin{tabular}{|c|c|c|}
\hline & Endogenous metabolites & Exogenous drugs \\
\hline \multirow[t]{4}{*}{ Complex lipid } & Glycerophospholipids; & \multirow{9}{*}{$\begin{array}{l}\text { e.g. olanzapine (Antipsychotic), }{ }^{2,}{ }^{25)} \\
\text { imatinib (Cancer drug), }{ }^{25)} \\
\text { vinblastine (Cancer drug), }{ }^{26)} \\
\text { banoxantrone (Cancer drug) }{ }^{27)} \\
\text { diazepam }{ }^{28)} \text { and temazepam } \\
\text { (Antianxiety drugs, Hypnotic) }^{28}\end{array}$} \\
\hline & 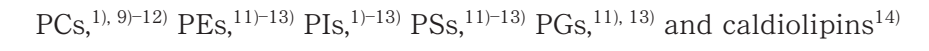 & \\
\hline & Glycosphingolipids & \\
\hline & Gangliosides, ${ }^{15), 16)}$ sulfatides, ${ }^{13), 16), 17)}$ and galactocyl-ceramide ${ }^{18), 19)}$ & \\
\hline \multirow[t]{5}{*}{ Simple lipid } & Neutral lipids; & \\
\hline & triacylglycerols, ${ }^{12)}$ diacylglycerols, ${ }^{12)}$ and cholesterol ${ }^{11,20)}$ & \\
\hline & Fatty acids $^{21)}$ & \\
\hline & Other metabolites; & \\
\hline & Amino acids ${ }^{54)}$ flavonoids, $\left.{ }^{21,}, 54\right)$ oligosaccharides, $\left.{ }^{21,}, 54\right)$ and heme ${ }^{23), 24)}$ & \\
\hline
\end{tabular}

Note: $\mathrm{PC}=$ phosphatidylcholine, $\mathrm{PE}=$ phosphatidylethanolamine, $\mathrm{PI}=$ phosphatidylinositol, $\mathrm{PS}=$ phosphatidylserine, $\mathrm{PG}=$ phosphatidyglycerol.

munohistochemistry based on antibodies. In this context, metabolic imaging by IMS is an important tool for interpretation of various aspects of life science (Table 1).

IMS as a metabolite-imaging tool in tissues or cells has the following advantages: First, IMS does not require any labels or specific probes; second, IMS is a non-targeted imaging method, thus, we can localize unexpected metabolites; finally, the simultaneous imaging of many types of metabolite molecules is possible. Given the enormous molecular diversity of metabolite species, a large number of which are still unknown, all these features are necessary for the assessment of metabolite localization. In addition, using tandem MS analysis, the detailed structure of metabolite molecules can be identified directly on the tissue sections; thus, it can be confirmed that the observed mass signals are derived from the molecules of interest. 1), 8) This technique is also effective for discriminating isobaric ions. ${ }^{1), 2)}$

\section{Sample Preparation Strategy of IMS for Small Organic Compounds}

Today, the application of IMS to researching small organic compounds $(m / z<1000)$ can be subdivided into two distinct areas: (1) measurement of endogenous compounds (such as lipids), and (2) measurement of exogenous compounds (such as drugs).

As endogenous metabolites, lipids have been intensively investigated. Studies describing detection and imaging of several types of lipids, namely, complex lipids (e.g., glycerol-phospholipids ${ }^{1,9)-14)}$ and glycosphingolipids ${ }^{13), 15)-19)}$ ) and simple lipids (e.g., cholesterol, ${ }^{11,20)}$ acylglycerides, ${ }^{12)}$ and fatty acids $^{21)}$ ) have been reported. In addition, other metabolites with superior ionization efficiency, such as heme, which is a prosthetic group that consists of an iron atom contained in the center of a porphyrin ring, interestingly, even in African ritual art object, ${ }^{22}$ have also been investigated $^{23), 24)}$ (Table 2).

Mapping of administered drug metabolism and its metabolite distribution by IMS is also attracting a lot of attention because of advantages over conventional imaging techniques, as will be discussed subsequently. In this area, so far, a number of studies regarding antipsychotics, ${ }^{2), 25)}$ cancer drugs, ${ }^{25)-27)}$ antianxiety drugs, and hypnotics ${ }^{27)}{ }^{28)}$ have been reported (Table 2 ). We shall introduce a representative research application in imaging both endogenous and exogenous metabolites.

\section{IMS for Endogenous Compounds: Phospholipids}

MALDI-IMS is effective for profiling ${ }^{10), 22), 29)}$ and visualizing distribution ${ }^{1), 30)-32)}$ of endogenous lipids such as glycerophospholipids; they can also be imaged with SIMS. ${ }^{33)-36)}$ For example, when a mouse brain section treated with 2,5-dihydroxybenzoic acid (DHB) solution was immediately analyzed by MS, strong peaks that are mainly derived from phospholipids were observed between mass region of $400<m / z<900$ (Fig. $2)^{9), 10), 13)}$ This is because phospholipids are ionized efficiently due to the following reasons; first, large amounts - more than $60 \%$ in dry weight - of the brain tissue consists of lipids. Second, these compounds have an easily ionizable structure; phospholipids, in particular, phosphatidylcholines [PCs] contain a phosphate group and trimethylamine that are easily charged. ${ }^{37)}$

Glycerophospholipids comprise a large molecular 


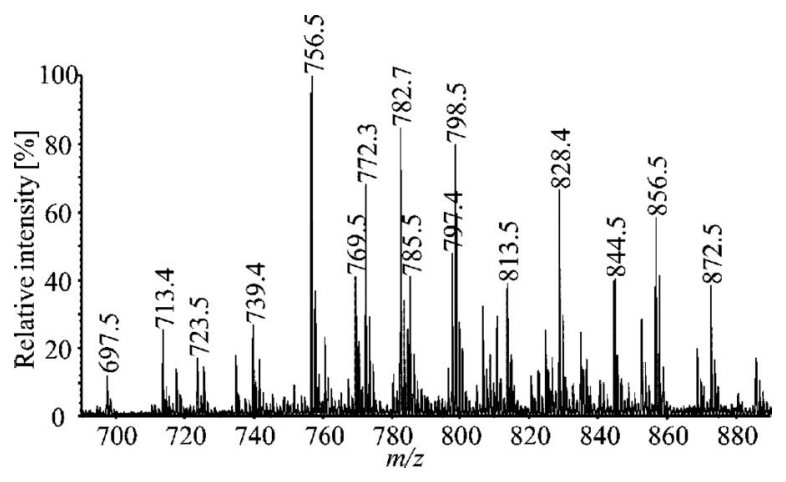

Fig. 2. Mass spectrum obtained from a mouse retinal section. The mouse retinal sections under dark adaptation were sprayed with a 2,5-dihydroxybenzoic acid (DHB) solution as a matrix. After drying, the sections were raster-scanned using MALDI-quadrupole ion trap (QIT)- time-of-flight (TOF)-based IMS. The MS data was then accumulated and averaged from a single raster scan. Sixteen ions are discussed in this study, and the values of $m / z$ are indicated over each ion in the mass spectrum.

(Reprinted from ref. 9, with permission from John Wiley \& Sons, Ltd., copyright 2008.)

(a)<smiles>[X]C(=O)OOC[C@H](COC)COC(C)=O</smiles>

(b)

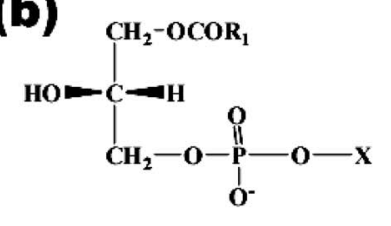

family in which phosphoric acid is bound via ester formation to a glycerolipid (Fig. 3). They are subdivided into distinct classes (e.g., PCs, phosphatidylethanolamines [PEs], and phosphatidylinositols [PIs]) based on the structure of the head group linked to the phosphate, attached at the $s n-3$ position of the glycerol backbone. They are further subdivided into numerous molecular species on the basis of the composition of the fatty acids linked to the $s n-1$ and $s n$ - 2 positions of the glycerol backbone. Using IMS, we can image not only these multiple classes, but also related molecular species simultaneously. In particular, capability of IMS to determine the distinct localization of each molecular species, in other words, elucidation of distinct fatty acid composition of biological membranes in different tissue locations, is an important advantage of IMS (Fig. 4). The reason is that since several types of fatty acids, especially polyunsaturated fatty acids (PUFA), in the phospholipids are released and converted into bioactive lipids in response to extracellular stimuli, which mediate various important biological processes ${ }^{38}$ thus distinct distribution information of PUFA-containing molecular species is quite valuable. ${ }^{39)}$ Figure 5 shows the distribution of distinct PC molecular species in mouse retinal tissue, which have multilayered structures of approximately $150 \mu \mathrm{m}$ thickness. MALDI-IMS successfully revealed the layer-specific distribution of

(c)

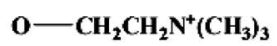

\section{phosphatidylcholine}

$$
\mathrm{O}-\mathrm{CH}_{2} \mathrm{CH}_{2} \mathrm{~N}^{+} \mathrm{H}_{3}
$$

\section{phosphatidylethanolamine}

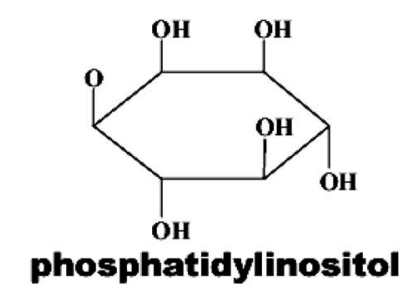

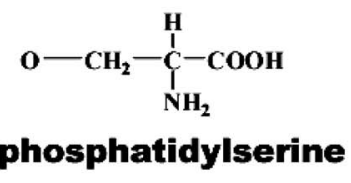<smiles>C[C@H](CO)[C@H](C)CO</smiles>

phosphatidylglycerol

Fig. 3. Structure of phospholipid classes.

Structures of the glycerol backbone of phospholipids (a) and lyso-phospholipids (b) are shown. They are subdivided into distinct classes (e.g., phosphatidylcholines, phosphatidylethanolamines, and phosphatidylinositols) based on the structure of the head group linked to the phosphate, attached at the $s n$ - 3 position of the glycerol backbone (c). They are further subdivided into numerous molecular species on the basis of the composition of fatty acids linked to the $s n-1$ and $s n-2$ positions of the glycerol backbone. 


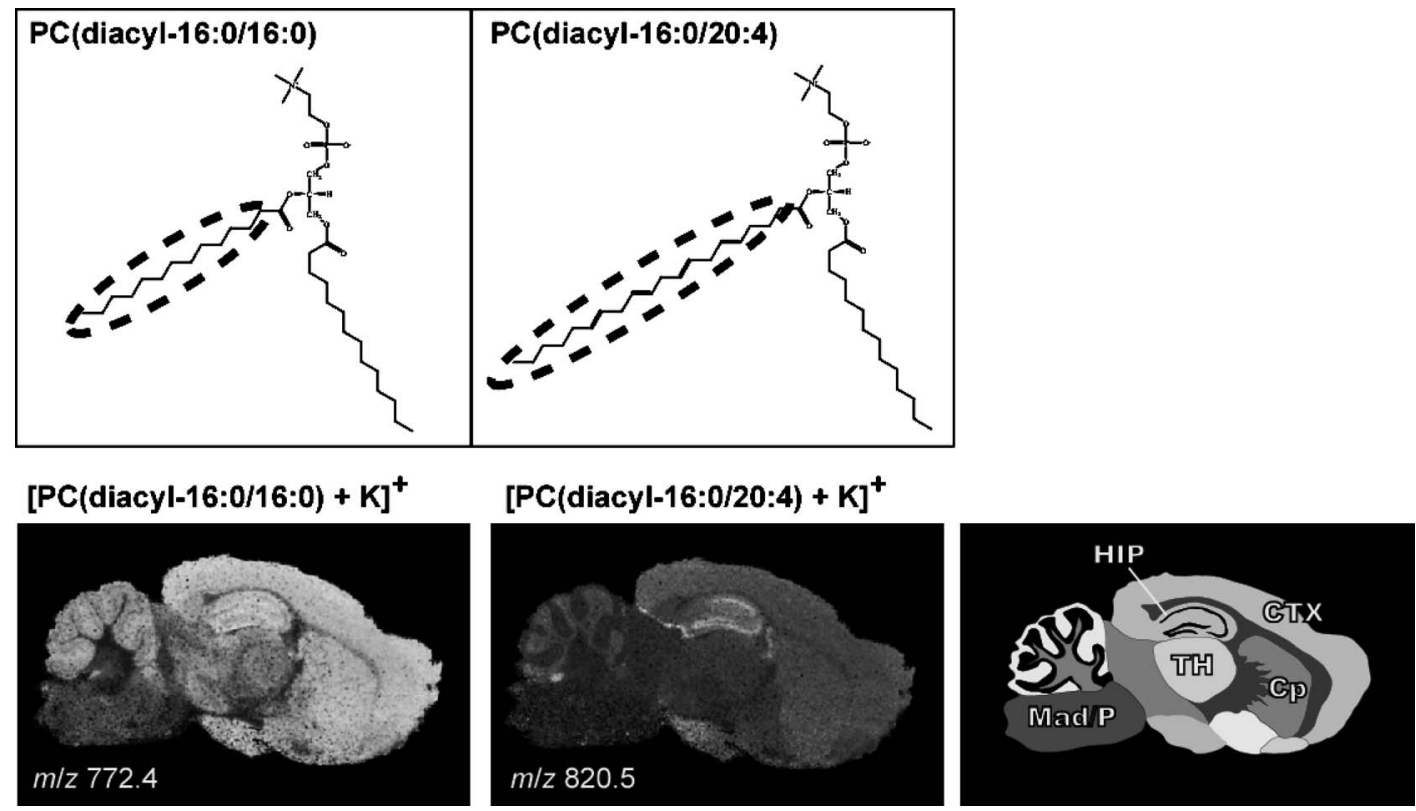

Fig. 4. Distinct localization of phospholipid molecular species revealed by IMS.

Different distribution pattern of phospholipids arise from the distinct fatty acid composition of glycerophospholipids.

PC molecular species, including PUFA containing PCs (Fig. 5). ${ }^{9)}$ Currently, only IMS can visualize such different structures of phospholipids that are hidden in the cellular membrane, therefore, imaging of individual phospholipid molecular species with IMS will greatly contribute to the field of lipid biochemistry.

\section{IMS for Endogenous Compounds: Gangliosides}

Gangliosides are glycosphingolipids consisting of mono- to polysialylated oligosaccharide chains of variable lengths attached to a ceramide unit. They are inserted in the outer layer of the plasma membrane, with the hydrophobic ceramide moiety acting as an anchor, while their oligosaccharide moiety is exposed to the external medium. ${ }^{40)}$ Gangliosides also comprise a large family; their constituent oligosaccharides differ in the glycosidic linkage position, sugar configuration, and the contents of neutral sugars and sialic acid. Along with the oligosaccharide unit, the ceramide moiety of gangliosides also varies with respect to the type of long-chain base (LCB) (sphingosine base) and the fatty acid moiety to which it is coupled (Fig. 6).

Previous biochemical studies have revealed that the LCB of the brain ganglioside species has either 18 or 20 carbon atoms (i.e., C18- or C20-sphingosine), and C20sphingosine (C20-LCB species) is present in significant amounts only in the central nervous system. ${ }^{41-44)}$ Its content increases significantly in rodents and humans throughout life. ${ }^{45)-47)}$ The C20-LCB gangliosides are of great interest because of their characteristic brain specificity and their dramatic increase during the organism's lifespan. However, lack of visualization technology for specific detection and visualization of $\mathrm{C} 18$ and $\mathrm{C} 20$ gangliosides has left us incapable of determining their precise tissue distribution. Antibodies to some oligosaccharide moieties are available for visualizing the molecular species with different constituent oligosaccharides, ${ }^{48)}$ but such immunologi- cal methods cannot detect the differences in the ceramide structure, which is hidden in the lipid bilayer.

Due to the negative charge on the sialic acids and their rich abundance in the brain, gangliosides are strongly detectable in the $1,500<m / z<2,500$ range with IMS in the negative ion detection mode ${ }^{11), 15), 16)}$ (Table 3). In addition, IMS discriminates not only structural differences in oligosaccharides but also in the lipid moiety, and therefore, successfully reveals the specific distribution of the C20-LCB species in the mouse brain. While the $\mathrm{C} 18$ species is widely distributed throughout the frontal brain, the C20 species is selectively localized in specific brain regions, namely, in the molecular layer (ML) of the dentate gyrus (DG). Furthermore, the developmental- and aging-related accumulation of the C20 species in the ML-DG can be visualized, ${ }^{15)}$ i.e., we could specify the tissue location where C20 gangliosides accumulate (Fig. 7). These observations indicate that this brain region-specific regulation of LCB chain length is, in particular, important for its distinct function in the brain. As this study clearly demonstrates, the novel capabilities of IMS could shed light on long-standing questions in the biological/clinical field.

\section{IMS for Exogenous Drugs}

An important phase of drug discovery is determining how a drug-candidate compound is distributed and metabolized within the body. The use of IMS to monitor drug delivery has also attracted much interest. Compared to traditional whole-body autoradiography (WBA) using radio-labeled compounds, IMS offers many advantages in the determination of drug distribution. First, we can simultaneously and discriminately monitor not only the intact drug molecules, but also their metabolites; ;), 25), 26), 28) WBA cannot distinguish these molecules. Thus, IMS can determine whether medicinally intact drugs have reached the 
(a)

(c)

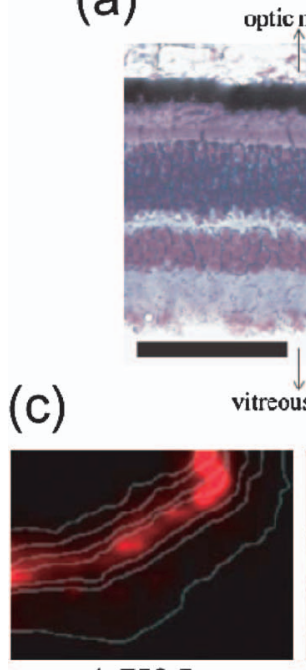

(b)

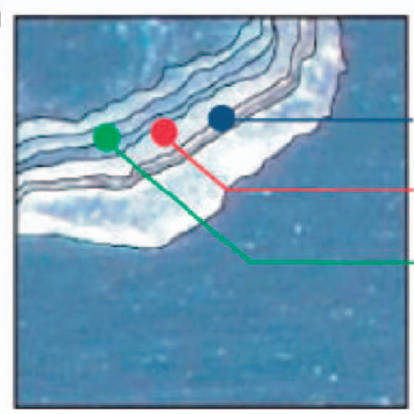

pigment epithelium

outer segments

inner segments

outer nuclear layer

outer plexiform layer

inner nuclear layer $\mathrm{m} / \mathrm{z} 756.5$

$m / 2782.7$

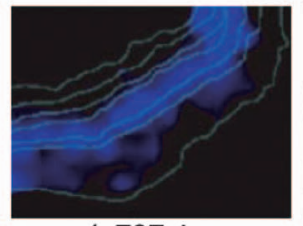

$\mathrm{m} / \mathrm{z} 797.4$

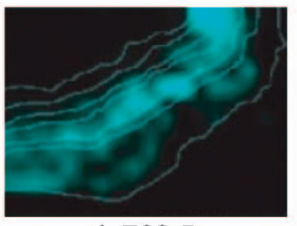

$m / 2769.5$

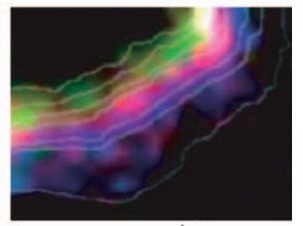

merged

Fig. 5. Distribution of $\mathrm{PC}$ molecular species in a mouse retinal section.

A. (a) The retinal layer structure can be roughly distinguished in this optical image of the mouse retinal section, and is indicated as three colored dots. (b) The ion image merged from 4 different ions of PC molecular species revealed the three-zone distribution of the retinal section.

(Reprinted from ref. 9, with permission from John Wiley \& Sons, Ltd., copyright 2008.)
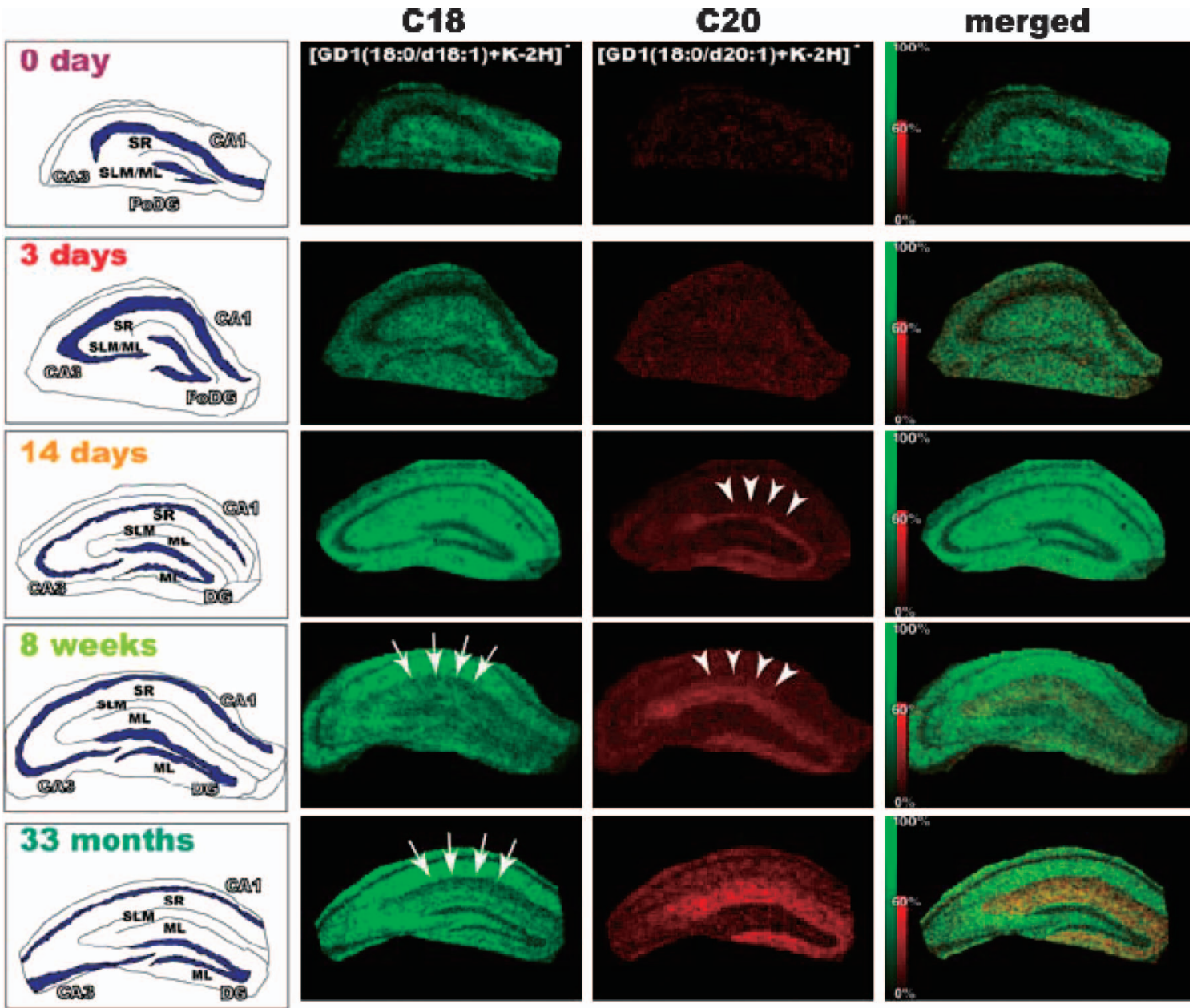

Fig. 7. Developmental- and aging-related accumulation of C20-GD1 in the ML and stratum lacunosum molecular (SLM) of the hippocampal formation.

Visualization of the ion corresponding to GD1 $(\mathrm{m} / z 1,874$ and 1,902$)$ in the mouse hippocampus at the indicated timepoints (postnatal 0, 3, and 14 days, and 1 and 33 months). In the P14 mouse hippocampus, C20-GD1 was concentrated in the narrow area of DG-SMm and began to spread over the medial edge of the region (arrow heads). In contrast, the concentration of the $\mathrm{C} 18$ species decreased in the ML/SLM with aging (arrows).

(Reprinted from ref. 15.) 


\section{C18-species}

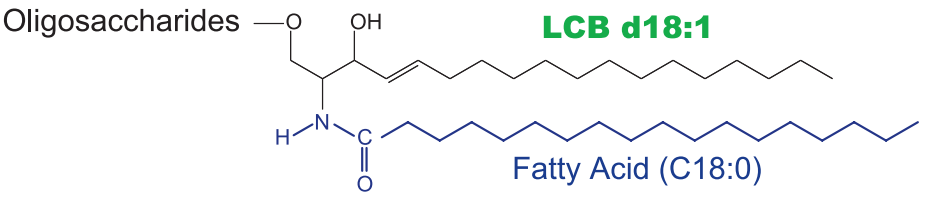

\section{C20-species}

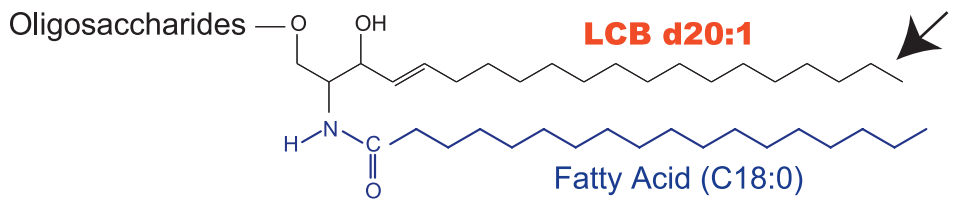

Fig. 6. Structures of ganglioside molecular species containing C18-long-chain backbone (LCB) and C20-LCB. The C20 species has 2 more carbon atoms in its LCB moiety than does the C18 species (arrow).

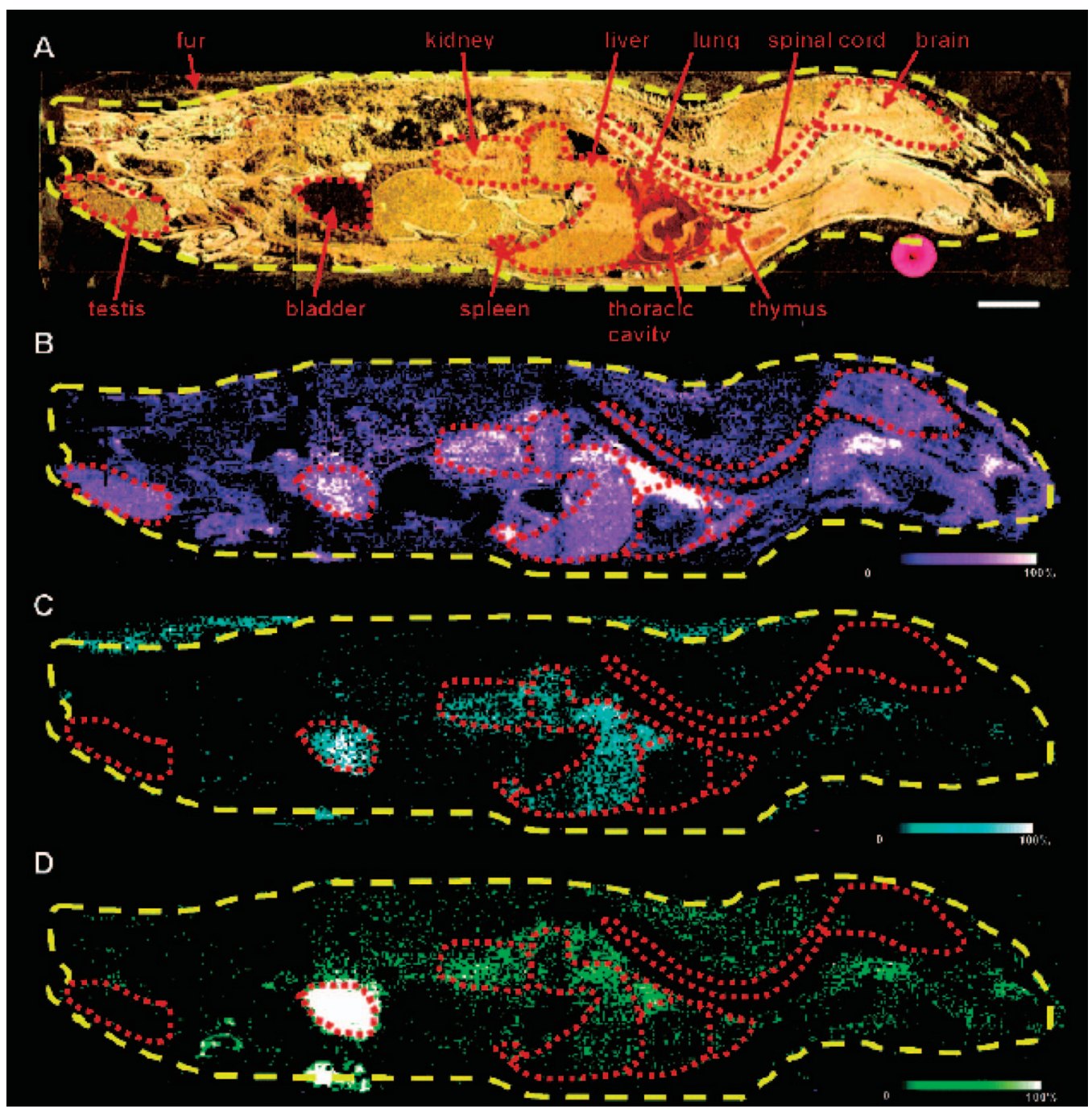

Fig. 8. Detection of drug olanzapine and its metabolite distribution in a whole-body sagittal tissue section by single IMS analysis.

Optical image of a $2 \mathrm{~h}$ post olanzapine-dosed rat tissue section across four gold MALDI target plates (A). Organs are outlined in red, and pink dot is used as a timepoint label. MS/MS ion image of olanzapine $(\mathrm{m} / z$ 256) (B). MS/ MS ion image of $N$-desmethyl metabolite $(\mathrm{m} / z$ 256) (C). MS/MS ion image of 2-hydroxymethyl metabolite $(\mathrm{m} / z$ 272) (D). Bar denotes $1 \mathrm{~cm}^{2}{ }^{2}$

(Reprinted from ref. 2, with permission from American Chemical Society, copyright 2006.) 
Table 3. Detection of Gangliosides in Mouse Hippocampus

\begin{tabular}{lcccccc}
\hline & \multicolumn{5}{c}{ Negative ions } \\
\hline & {$[\mathrm{M}-\mathrm{H}]^{-}$} & {$[\mathrm{M}+\mathrm{Na}-2 \mathrm{H}]^{-}$} & {$[\mathrm{M}+\mathrm{K}-2 \mathrm{H}]^{-}$} & {$[\mathrm{M}+2 \mathrm{Na}-3 \mathrm{H}]^{-}$} & {$[\mathrm{M}+\mathrm{Na}+\mathrm{K}-3 \mathrm{H}]^{-}$} & {$[\mathrm{M}+2 \mathrm{~K}-3 \mathrm{H}]^{-}$} \\
\hline GM1 $(\mathrm{d} 18: 1 / 18: 0)$ & 1,544 & - & - & - & - & - \\
GM1 $(\mathrm{d} 20: 1 / 18: 0)$ & 1,572 & - & - & - & - \\
\hline GD1 $(\mathrm{d} 18: 1 / 18: 0)$ & - & 1,858 & 1,874 & - & - & - \\
GD1 $(\mathrm{d} 20: 1 / 18: 0)$ & - & 1,886 & 1,902 & - & 2,186 & 2,202 \\
\hline GT1 $(\mathrm{d} 18: 1 / 18: 0)$ & - & - & - & 2,170 & 2,214 & 2,230 \\
GT1 $(\mathrm{d} 20: 1 / 18: 0)$ & - & - & - & 2,198 & - \\
\hline
\end{tabular}

Table 4. Current Limitation and Perspective of IMS for Small Molecules

\begin{tabular}{|c|c|c|}
\hline $\begin{array}{l}\text { Limitation } \\
\text { process }\end{array}$ & Problems & Suggested solutions \\
\hline Ionization & $\begin{array}{l}\text { High ionization efficiency of the targeted } \\
\text { molecule is required. }\end{array}$ & $\begin{array}{l}\text { Optimization of sample preparation procedure }{ }^{59)} \\
\text { Discovery and development of novel matrices }{ }^{12), 30)} \text { and } \\
\text { ionization method }{ }^{19), 53), 54}\end{array}$ \\
\hline Ion-separation & $\begin{array}{l}\text { Multiple compounds often share the same } \\
\text { nominal mass in low } m / z \text { region. }\end{array}$ & $\begin{array}{l}\text { Imaging in tandem MS mode }{ }^{1,2)} / \text { ion-separation with } \\
\text { ion-mobility cell }{ }^{30), 31)} / \text { imaging with FTICR-instrument } \\
\text { development of organic-matrix free ionization method } \\
\left(\text { such as use of nanoparticles, }{ }^{19), 31)} \text { ME-SALDI, }{ }^{55)} \text { DIOS, }\right. \\
\text { and NIMS }\end{array}$ \\
\hline $\begin{array}{l}\text { Spatial } \\
\text { resolution }\end{array}$ & $\begin{array}{l}\text { Challenge for the sub-cellular spatial resolution } \\
(>10 \mu \mathrm{m}) \text {. }\end{array}$ & $\begin{array}{l}\text { Improvement in instrument, }{ }^{60)} \text { such as 2-dimensional } \\
\text { detector }^{61)} / \text { use of nanoparticles }^{19)}\end{array}$ \\
\hline
\end{tabular}

target organs or not. Second, IMS can visualize the distribution of drugs at a lower cost and in a much shorter time compared with detection using isotopes. ${ }^{49)}$

Figure 8 reveals the detection of drugs that have been delivered orally in mice. ${ }^{2)}$ Khatib-Shahidi et al. have successfully investigated the distribution of the antipsychotic drug olanzapine and its metabolites in a whole rat sagittal section $6 \mathrm{~h}$ after administering the dose. This study clearly showed the distinct distribution of intact drugs and their metabolites; the intact drug reached the target organ (the brain), whereas its metabolites were localized in the bladder.

\section{Current Limitations and Perspective}

Despite the promising capability of IMS for imaging small metabolites, problems still remain in this emerging technique in each step of the measurement (summarized in Table 4). We now discuss each of the problems.

(1) It is necessary to achieve high ionization efficiency of the targeted molecule

In MALDI-IMS, in which the tissue sample is measured directly, one should pay attention to the fact that samples are an extremely complex mixture of biomolecules. Since tissues and cells are the subject of MALDI-IMS, the sample clean-up procedure is limited, whereas in traditional MS research, analyte molecules are generally extracted and separated from crude samples by gas chromatography or high-performance liquid chromatography (HPLC). When a crude sample is subjected to MS, numerous molecular species compete for ionization, and eventually, molecules that are easily ionized reach the detector preferentially while suppressing the ionization of other molecules, causing severe ion suppression effects. ${ }^{50)-52)}$ In fact, using a mouse whole-body section coated with analyte drugs, Stoeckli and colleagues have found small regions (although less than 5\% of the total region) in which the drugs could not be detected, presumably because of ion suppression effects. ${ }^{49)}$ Thus, an important point in an IMS experiment, especially in targeted analysis, is optimizing the sample condition so that the analyte molecule present in the crude mixture can be efficiently ionized, and sample preparation has a critical role. In particular, it is helpful to perform a preliminary experiment using a reference compound. The choice of a suitable matrix compound, as well as optimizing the composition of the matrix solution, can improve the ionization efficiency of the molecules of interest. Atkinson and colleagues have described an excellent example of this problem. They tried to visualize the distribution of an anti-cancer compound, AQ4N (1,4-bis- $\{[2$-(dimethylamino)ethyl]amino\}-5,8-dihydroxyanthracene-9,10-dione), and found that it is hardly ionized by itself because it forms complexes with DNA. By screening matrices, they found one that can support the ionization of $\mathrm{AQ} 4 \mathrm{~N}$ without generating complexes by raising the acidity of the matrix solution, and succeeded in visualizing the distribution of AQ4N (Fig. 9). ${ }^{27)}$ Thus, important tasks for the future are discovery and development of novel matrices ${ }^{12), 30)}$ and alternative ionization methods. ${ }^{19), 53), 54)}$ 


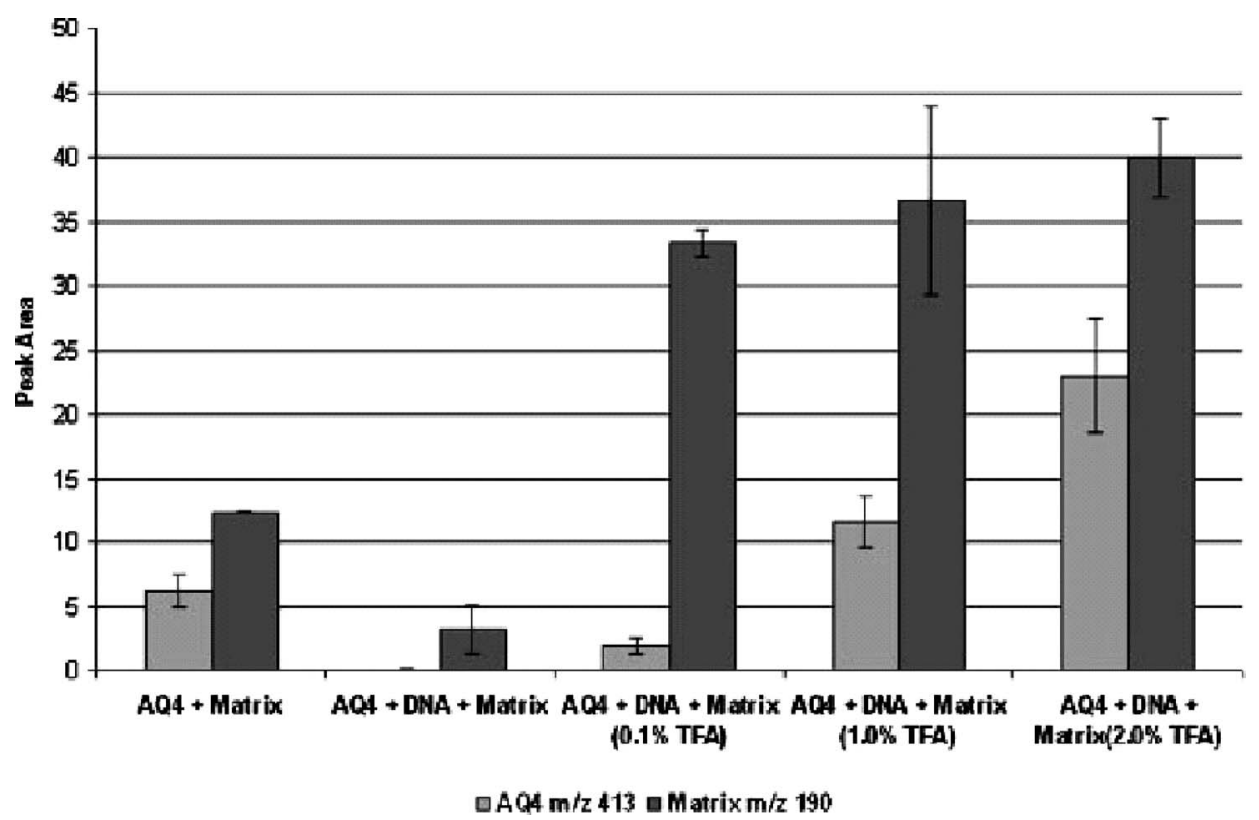

Fig. 9. Optimization of the condition of ionization for the drug banoxantrone (AQ4N).

Since AQ4 forms complexes with DNA, a high concentration of trifluoroacetic acid (TFA) was added to the matrix solution, to avoid complex formation.

(Reprinted from ref. 27, with permission from John Wiley \& Sons, Ltd., copyright 2007.)

(2) Development of analysis method to separate the multiple compounds that share the same nominal mass

The low- $m / z$ region $(<m / z 1000)$ of a MALDI spectrum contains a large population of ions from endogenous metabolites, as well as matrix-related adduct clusters and fragments. 1), 25) Due to such a high density of ions, multiple compounds often share the same nominal mass. In such a case, one of the solutions is to perform advanced MS analysis to distinguish target compounds of interest from this extensive chemical background. Several groups have demonstrated that imaging in the tandem-MS mode provides highly specific information about a target molecule. Essentially, all ions in the range of the targeted precursor are fragmented, and the abundance of the target compound is determined from the measured intensity of one or more of its structurally significant fragment ions. This measurement is performed in the tissue for each precursor of interest. ${ }^{1,2), 26)}$ By performing tandem MS-scanning in a positive ion detection mode on rat brain, Garret et al. demonstrated that a peak at $m / z$ 828 contains 3 types of ions: PC (diacyl-16:0/22:6), PS (diacyl-16:0/18:1), and a DHB cluster (Fig. 10). ${ }^{1}$ ) In addition, the combination of tandem MS and ion mobility separation adds a further separation step and provides more specific information. $\left.{ }^{26)}, 30\right), 31$ ) Another approach is to use MALDI-Fourier transform ion cyclotron resonance MS (MALDI-FTICR MS) for tissue imaging. ${ }^{25)}$ Cornett et al. showed that this technique utilizes its high resolving power to produce images from thousands of ions measured during a single MS pass. Accurate mass measurement provides high molecular specificity for the ion images on the basis of elemental composition. Figure 11 reveals FTICR images of hydroxymethyl-olanzapine and three isobaric ions. ${ }^{25)}$ This example clearly demonstrates that the four major ions are observed at the same nominal $\mathrm{m} / \mathrm{z}$ of the 2-hydroxymethyl metabolite of olanzapine (exact mass: $329.1431 \mathrm{u}$ ). Although they were detected within a range of only $0.02 \mathrm{u}$, these ions were sufficiently resolved by FTICR MS, and each ion exhibited a distinct ion distribution image (Fig. 11).

Furthermore, elimination of matrix-derived ions is effective for reducing the overlap of peaks from multiple compounds, thereby obtaining specific information in a targeted analysis. Several important studies have been published in which matrix-free ionization was developed, such as the use of nanoparticle-based ionization, ${ }^{19), 31)}$ matrix-enhanced surface-assisted laser desorption/ionization (ME-SALDI), ${ }^{55)}$ desorption/ionization on silicon (DIOS), ${ }^{56)}$ and nanostructure-initiator mass spectrometry (NIMS). ${ }^{57)}$

(3) Challenges to achieve sub-cellular spatial resolution

Improvement of the spatial resolution toward subcellular resolution $(>10 \mu \mathrm{m})$ is another important challenge. Metabolite imaging within cellular organelles will provide valuable information about cellular metabolism. In this regard, imaging with SIMS has achieved spatial resolution of sub-micrometer order, and successfully visualized subcellular structures, ${ }^{34), 35)}$ and even visualized dynamic changes of metabolite molecules during Tetrahymena mating. ${ }^{34)}$ However, both soft ionization of analyte and tandem MS are difficult to achieve using typical SIMS techniques. ${ }^{58)}$ On the other hand, spatial resolution in MALDI-IMS is much lower than that of SIMS; it depends on the experimental conditions and instrumentation, but is typically 20$100 \mu \mathrm{m}$. Among the limitations in this technique are the size of the organic matrix crystal and the migration of analytes during the matrix application process. To overcome these limitations, Taira et al. reported a nanoparticle-assisted laser desorption/ionization 
(A)

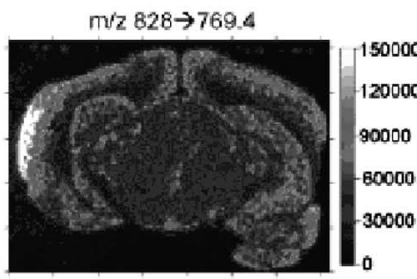

(B)

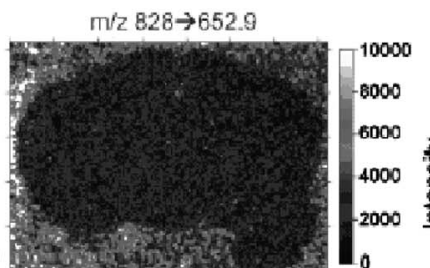

(C)

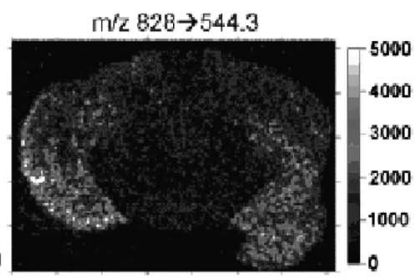

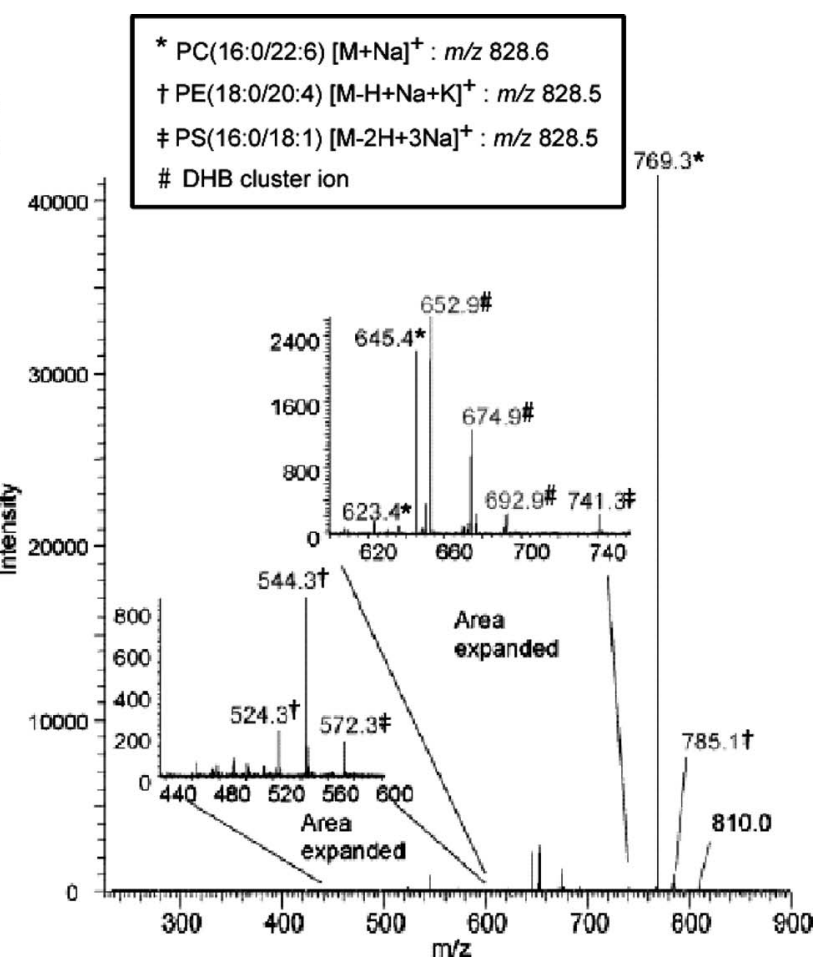

$\mathrm{m} / \mathrm{z}$

Fig. 10. An example of imaging in tandem MS mode, demonstrating that a peak at $\mathrm{m} / z 824$ contains at least 4 types of ions.

Average $\mathrm{MS}^{2}$ spectrum isolating for $\mathrm{m} / \mathrm{z}$ 828.6, and specific MS images for three product ions are shown as insets. The use of $\mathrm{MS}^{2}$ and $\mathrm{MS}^{3}$ enabled the identification of 4 different isobaric ions appearing at $\mathrm{m} / z 828.6$ with an isolation window of $1.5 \mathrm{u}$. The images generated from the MS/MS product ions of the isobars $(\mathrm{A}-\mathrm{C})$ show a different distribution for each isobar.

(Reprinted from ref. 1, with permission from Elsevier, copyright 2006.)

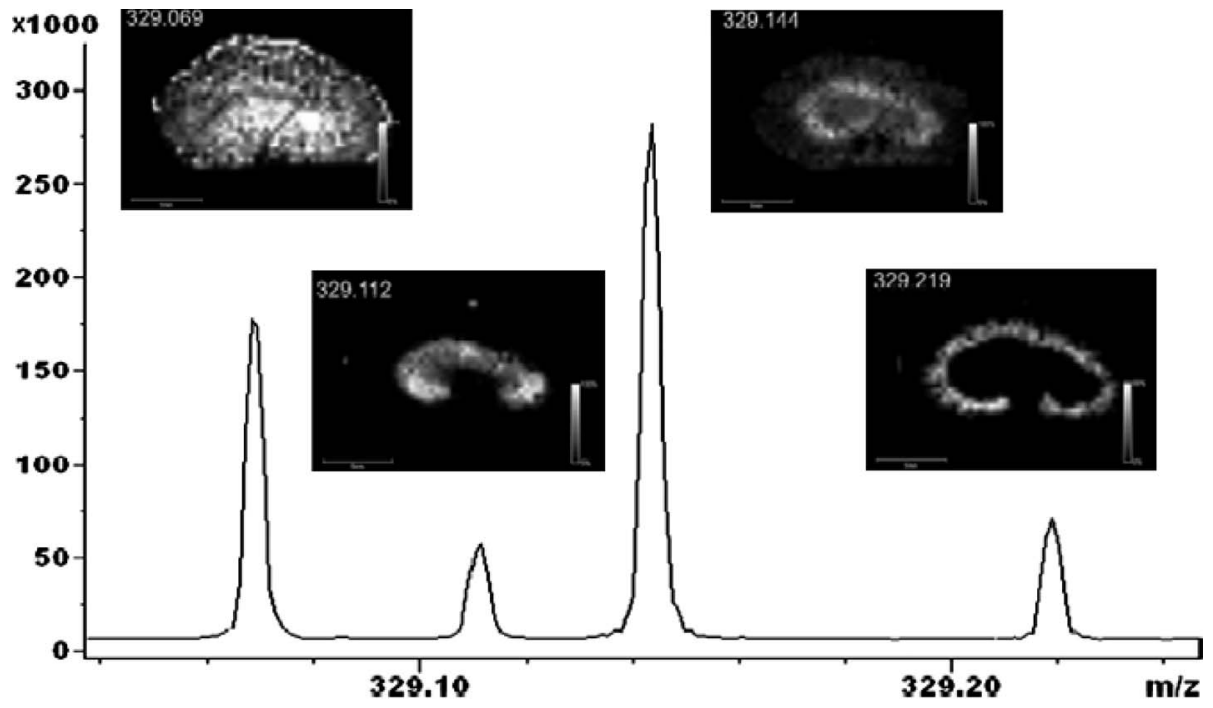

Fig. 11. MALDI-FTICR images of hydroxymethyl olanzapine and three isobaric ions.

The four major ions observed at the same nominal $\mathrm{m} / \mathrm{z}$ of the 2-hydroxymethyl metabolite of olanzapine (exact mass; $329.1431 \mathrm{u}$ ). Although they were detected within a range of only $0.02 \mathrm{u}$, these ions are sufficiently resolved by use of FTICR MS and each ion exhibited a distinct ion distribution image. (Reprinted from ref. 25, with permission from American Chemical Society, copyright 2008.)

(nano-PALDI)-based IMS, in which the matrix crystallization process is eliminated. Improved spatial resolution was achieved using a commercial mass spectrometer. $^{19)}$ Figure 12 shows the ability of nano-PALDI IMS to visualize lipid distribution within the internal structure of the mouse hippocampus. Application of this technique clearly revealed layer-structure-specific localization of sulfatides at a resolution of $15 \mu \mathrm{m} .{ }^{17)}$ Furthermore, by utilization of such organic-matrix free ionization for IMS, matrix-related signals can be excluded, therefore making the process useful especially for small molecule imaging. 

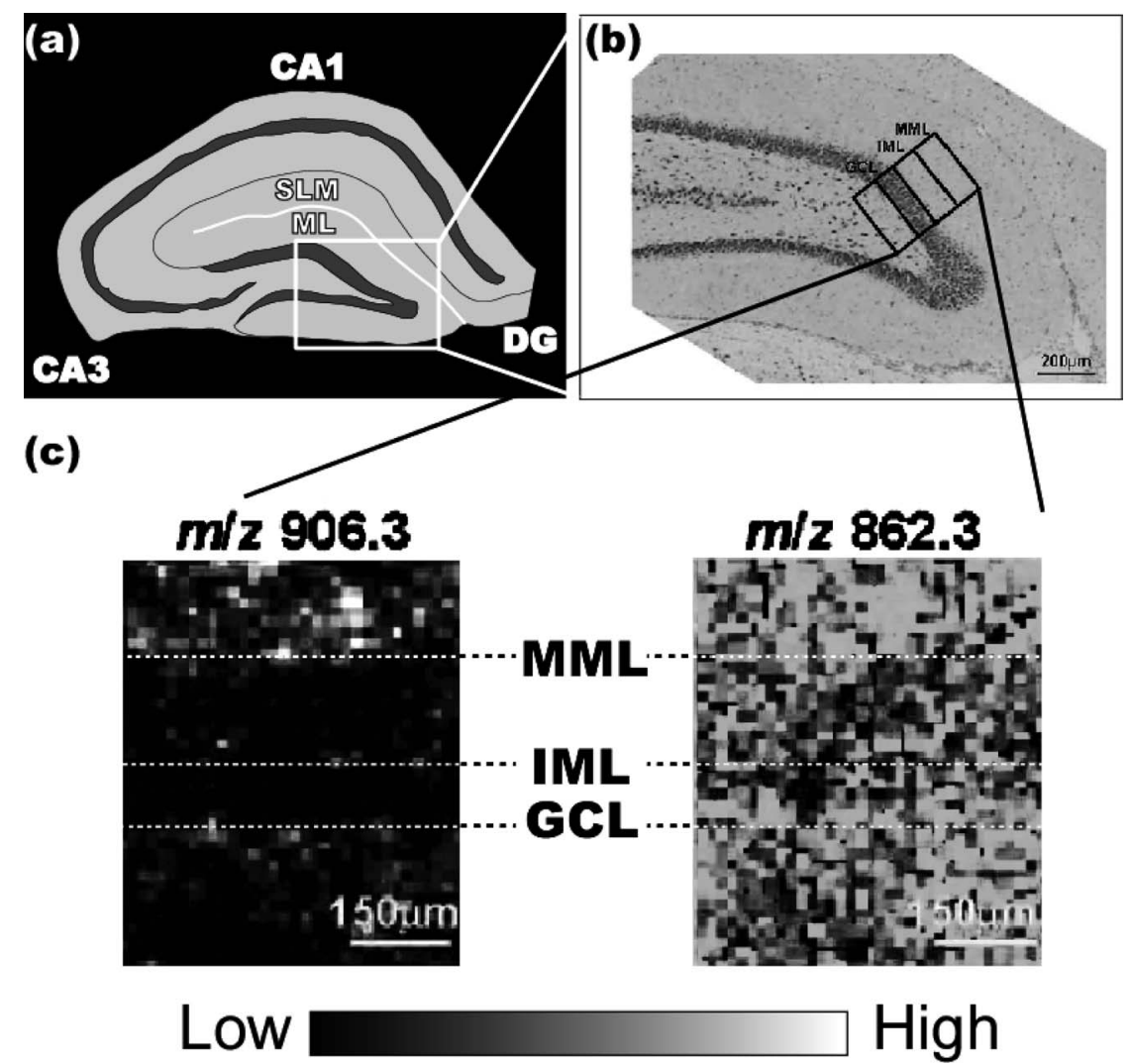

$m / 2862.3$

Fig. 12. nano-PALDI based imaging improves spatial resolution in IMS.

(a) Structure of rat hippocampus and (b) optical image of Nissle stained-rat hippocampus. GCL, granular cell layer; IML, inner molecular layers; MML, middle molecular layers. The black rectangle indicates the measurement area for nano-PALDI based IMS. The scale bar is $200 \mu \mathrm{m}$. (c) nano-PALDI imaging of lipid distributions for the dentate gyrus of the rat hippocampus. The scale bar is $150 \mu \mathrm{m}$. (Reprinted from ref. 17, with permission from Springer Japan, copyright 2008.)

\section{Conclusion}

IMS on small molecules has opened a new frontier in biological and clinical fields. Metabolite molecules such as lipids and other low molecular weight compounds in tissue sections cannot be observed with conventional light microscopic and electron microscopic techniques. As we describe in this article, the research field of metabolite imaging with MS is expanding. We expect it will become a standard method of reviewing metabolic distributions, and laboratories will be equipped with both IMS and traditional light microscopes in the near future as the IMS technique is simple and easy.

Herein, we have discussed the promising capabilities of IMS, as well as the importance of the experimental protocol, in particular, for the sample preparation step. As introduced here, with attention paid to some technical points, MALDI-IMS provides valuable information that could not be obtained by any other existing techniques; high selectivity, rapid acquisition, and parallel acquisition of multiple analytes. We expect that continuous improvement in the experimental protocol, as well as in the MS instrumentation, will further expand the capability of this emerging technique. In conclusion, we hope this review will help the readers to explore IMS as a new frontier in their research fields.

\section{References}

1) T. J. Garrett, M. C. Prieto-Conaway, V. Kovtoun, H. Bui, N. Izgarian, G. Stafford, and R. A. Yost, Int. J. Mass Spectrom., 260, 11 (2006).

2) S. Khatib-Shahidi, M. Andersson, J. L. Herman, T. A. Gillespie, and R. M. Caprioli, Anal. Chem., 78, 6448 (2006).

3) M. Stoeckli, D. Staab, M. Staufenbiel, K. H. Wiederhold, and L. Signor, Anal. Biochem., 311, 33 (2002).

4) P. Chaurand, J. L. Norris, D. S. Cornett, J. A. Mobley, and R. M. Caprioli, J. Proteome Res., 5, 2889 (2006).

5) R. M. Caprioli, T. B. Farmer, and J. Gile, Anal. Chem., 69, 4751 (1997).

6) P. Chaurand, M. Stoeckli, and R. M. Caprioli, Anal. Chem., 71, 5263 (1999).

7) M. Stoeckli, P. Chaurand, D. E. Hallahan, and R. M. Caprioli, Nat. Med., 7, 493 (2001).

8) S. Shimma, Y. Sugiura, T. Hayasaka, N. Zaima, M. Matsumoto, and M. Setou, Anal. Chem., 80, 878 (2008).

9) T. Hayasaka, N. Goto-Inoue, Y. Sugiura, N. Zaima, H. Nakanishi, K. Ohishi, S. Nakanishi, T. Naito, R. Taguchi, and M. Setou, Rapid Commun. Mass Spectrom., 22, 3415 (2008).

10) S. N. Jackson, H. Y. Wang, and A. S. Woods, J. Am. Soc. Mass Spectrom., 16, 2052 (2005).

11) S. N. Jackson, H. Y. Wang, and A. S. Woods, Anal. Chem., 77, 4523 (2005).

12) E. Astigarraga, G. Barreda-Gomez, L. Lombardero, O. 
Fresnedo, F. Castano, M. T. Giralt, B. Ochoa, R. Rodriguez-Puertas, and J.A. Fernandez, Anal. Chem., 80, 9105 (2008).

13) S. N. Jackson, H. Y. Wang, and A. S. Woods, J. Am. Soc. Mass Spectrom., 18, 17 (2007).

14) H. Y. Wang, S. N. Jackson, and A. S. Woods, J. Am. Soc. Mass Spectrom., 18, 567 (2007).

15) Y. Sugiura, S. Shimma, Y. Konishi, M. K. Yamada, and M. Setou, PLoS ONE, 3, e3232 (2008).

16) Y. Chen, J. Allegood, Y. Liu, E. Wang, B. CachonGonzalez, T. M. Cox, A. H. Merrill, Jr., and M. C. Sullards, Anal. Chem., 80, 2780 (2008).

17) H. Ageta, S. Asai, Y. Sugiura, N. Goto-Inoue, N. Zaima, and M. Setou, Medical Molecular Morphology, in press (2008).

18) S. Cha and E. S. Yeung, Anal. Chem., 79, 2373 (2007).

19) S. Taira, Y. Sugiura, S. Moritake, S. Shimma, Y. Ichiyanagi, and M. Setou, Anal. Chem., 80, 4761 (2008).

20) A. F. Altelaar, I. Klinkert, K. Jalink, R. P. de Lange, R. A. Adan, R. M. Heeren, and S. R. Piersma, Anal. Chem., 78, 734 (2006).

21) H. Zhang, S. Cha, and E. S. Yeung, Anal. Chem., 79, 6575 (2007).

22) V. Mazel, P. Richardin, D. Debois, D. Touboul, M. Cotte, A. Brunelle, P. Walter, and O. Laprevote, Anal. Chem., 79, 9253 (2007).

23) J. J. Jones, S. Borgmann, C. L. Wilkins, and R. M. O'Brien, Anal. Chem., 78, 3062 (2006).

24) S. Shimma and M. Setou, J. Mass Spectrom. Soc. Jpn., 55, $145(2007)$

25) D. S. Cornett, S. L. Frappier, and R. M. Caprioli, Anal. Chem., 80, 5648 (2008).

26) P. J. Trim, C. M. Henson, J. L. Avery, A. McEwen, M. F. Snel, E. Claude, P. S. Marshall, A. West, A. P. Princivalle, and M. R. Clench, Anal. Chem., 80, 8628 (2008).

27) S. J. Atkinson, P. M. Loadman, C. Sutton, L. H. Patterson, and M. R. Clench, Rapid Commun. Mass Spectrom., 21, 1271 (2007).

28) T. Kokaji, "Mass Microscopy; Imaging Mass Spectrometry Protocol Book," ed. by M. Setou, Springer Japan, Tokyo (2008), Chap. 7, p. 153.

29) M. Rujoi, R. Estrada, and M. C. Yappert, Anal. Chem., 76, 1657 (2004).

30) J. A. McLean, W. B. Ridenour, and R. M. Caprioli, J. Mass Spectrom., 42, 1099 (2007).

31) S. N. Jackson, M. Ugarov, T. Egan, J. D. Post, D. Langlais, J. A. Schultz, and A. S. Woods, J. Mass Spectrom., 42, 1093 (2007).

32) T. Hayasaka, N. Goto-Inoue, Y. Sugiura, N. Zaima, H. Nakanishi, K. Ohishi, S. Nakanishi, T. Naito, R. Taguchi, and M. Setou, Rapid Commun. Mass Spectrom., 22, 3415 (2008).

33) T. L. Colliver, C. L. Brummel, M. L. Pacholski, F. D. Swanek, A. G. Ewing, and N. Winograd, Anal. Chem., 69, 2225 (1997).

34) S. G. Ostrowski, C. T. Van Bell, N. Winograd, and A. G. Ewing, Science, 305, 71 (2004).

35) E. B. Monroe, J. C. Jurchen, J. Lee, S. S. Rubakhin, and J.
V. Sweedler, J. Am. Chem. Soc., 127, 12152 (2005).

36) D. Touboul, A. Brunelle, F. Halgand, S. De La Porte, and O. Laprevote, J. Lipid Res., 46, 1388 (2005).

37) M. Pulfer and R. C. Murphy, Mass Spectrom. Rev., 22, 332 (2003).

38) M. Murakami, Y. Nakatani, G. Atsumi, K. Inoue, and I. Kudo, Crit. Rev. Immunol., 17, 225 (1997).

39) D. Piomelli, G. Astarita, and R. Rapaka, Nat. Rev. Neurosci., 8, 743 (2007).

40) G. van Echten and K. Sandhoff, J. Biol. Chem., 268, 5341 (1993).

41) S. Sonnino and V. Chigorno, Biochim. Biophys. Acta, 1469, 63 (2000).

42) K. Sambasivarao and R. H. McCluer, J. Lipid Res., 15, 103 (1964).

43) H. P. Schwarz, I. Kostyk, A. Marmolejo, and C. Sarappa, J. Neurochem., 14, 91 (1967).

44) F. B. Jungalwala, V. Hayssen, J. M. Pasquini, and R. H. McCluer, J. Lipid Res., 20, 579 (1979).

45) P. Palestini, S. Sonnino, and G. Tettamanti, J. Neurochem., 56, 2048 (1991).

46) P. Palestini, M. Masserini, S. Sonnino, A. Giuliani, and G. Tettamanti, J. Neurochem., 54, 230 (1990).

47) J. E. Mansson, M. T. Vanier, and L. Svennerholm, J. Neurochem., 30, 273 (1978).

48) M. Kotani, I. Kawashima, H. Ozawa, T. Terashima, and T. Tai, Glycobiology, 3, 137 (1993).

49) M. Stoeckli, D. Staab, and A. Schweitzer, Int. J. Mass Spectrom., 260, 195 (2006).

50) E. Krause, H. Wenschuh, and P. R. Jungblut, Anal. Chem., 71, 4160 (1999).

51) F. Gharahdaghi, M. Kirchner, J. Fernandez, and S. M. Mische, Anal. Biochem., 233, 94 (1996).

52) T. M. Annesley, Clin. Chem., 49, 1041 (2003).

53) Y. Li, B. Shrestha, and A. Vertes, Anal. Chem., 79, 523 (2007).

54) Y. Li, B. Shrestha, and A. Vertes, Anal. Chem., 80, 407 (2008).

55) Q. Liu, Y. Xiao, C. Pagan-Miranda, Y. M. Chiu, and L. He, J. Am. Soc. Mass Spectrom., 20, 80 (2008).

56) Q. Liu, Z. Guo, and L. He, Anal. Chem., 79, 3535 (2007).

57) T. R. Northen, O. Yanes, M. T. Northen, D. Marrinucci, W. Uritboonthai, J. Apon, S. L. Golledge, A. Nordstrom, and G. Siuzdak, Nature, 449, 1033 (2007).

58) M. L. Kraft, P. K. Weber, M. L. Longo, I. D. Hutcheon, and S. G. Boxer, Science, 313, 1948 (2006).

59) B. Prideaux, S. J. Atkinson, V. A. Carolan, J. Morton, and M. R. Clench, Int. J. Mass Spectrom., 260, 243 (2007).

60) P. Chaurand, K. E. Schriver, and R. M. Caprioli, J. Mass Spectrom., 42, 476 (2007).

61) A. F. M. Altelaar, I. M. Taban, L. A. McDonnell, P. D. E. M. Verhaert, R. P. J. d. Lange, R. A. H. Adan, W. J. Mooi, R. M. A. Heeren, and S. R. Piersma, Int. J. Mass Spectrom., 260, 9 (2007)

Keywords: Imaging mass spectrometry, MALDI, Small molecules, Lipids, Drugs 